\title{
MASS DISTRIBUTION OF THE EO GALAXY NGC 6703 FROM ABSORPTION LINE PROFILE KINEMATICS
}

\author{
O.E. GERHARD \\ Astronomisches Institut, Venusstr. 7, CH-4102 Binningen \\ G. JESKE \\ Landessternwarte, Königstuhl, D-69117 Heidelberg \\ AND \\ R.P. SAGLIA AND RALF BENDER \\ Inst. für Astronomie, Scheinerstr. 1, D-816r9 München
}

Absorption line velocity profiles (vPs) contain important information on the anisotropy and mass distribution of elliptical galaxies (e.g., Gerhard 1993, Merritt 1993). Here we briefly present results of an extensive analysis of the E0 galaxy NGC 6703 (Gerhard et al. 1997). This work is part of an observational and theoretical program aimed at understanding the orbit structure and dark matter content of ellipticals at intermediate radii (a preliminary account is given in Saglia et al. 1997).

\section{Data and Analysis}

NGC 6703 is an E0 galaxy at a distance $D=36 h_{50}^{-1} \mathrm{Mpc}\left(h_{50} \equiv H_{0} / 50 \mathrm{~km} / \mathrm{s}\right.$ $/ \mathrm{Mpc}$ ). From a Jaffe profile fit, its absorption-corrected magnitude is $M_{\mathrm{B}}=$ -21.07 , or luminosity $L_{\mathrm{B}}=4.16 \times 10^{10} h_{50}^{-2} L_{\odot, \mathrm{B}}$. Its effective radius is $R_{\mathrm{e}}=$ $30^{\prime \prime}=5.2 h_{50}^{-1} \mathrm{kpc}$.

Our kinematic data for NGC 6703 extend to $2.6 R_{\mathrm{e}}$. The galaxy shows little rotation $\left(\approx 0 \mathrm{~km} / \mathrm{s}\right.$ for $R<R_{\mathrm{e}}, \approx 20-30 \mathrm{~km} / \mathrm{s}$ for $\left.R>R_{\mathrm{e}}\right)$. The velocity dispersion drops from the central $\approx 190 \mathrm{~km} / \mathrm{s}$ to $\approx 140 \mathrm{~km} / \mathrm{s}$ at $R_{e} / 2$, slowly declining to about $110 \mathrm{~km} / \mathrm{s}$ in the outer parts. The $h_{3}$ and $h_{4}$ values are everywhere close to zero.

With a new non-parametric technique we determine the DF $f\left(E, L^{2}\right)$ directly from the vP data. Monte Carlo tests using simulated data with the spatial extent, sampling, and error bars of the NGC 6703 data show that smooth DFs can be recovered to an RMS accuracy of $\sim 10 \%$, and the anisotropy parameter $\beta(r)$ to $\sim 0.1$, in a known potential. Similar tests analyzing models in different potentials show that, from data like ours for 


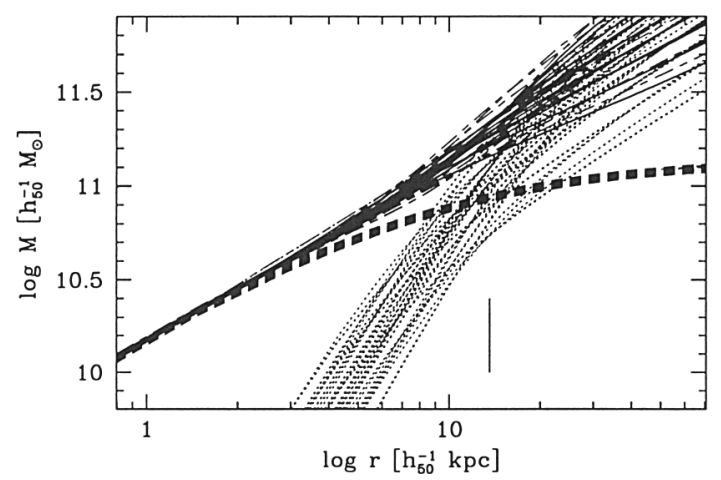

Figure 1. Luminous, dark, and total mass as a function of radius for acceptable models of NGC 6703 (short dashed, dotted, and dash-dashed or full lines, respectively). Mass distributions consistent with the data at $87 \%(1.5 \sigma)$ and at $95 \%$ c.l. $(2.0 \sigma)$ are coded by full and dash-dashed lines, respectively. The vertical line denotes the position of the last kinematic data point. At this radius, the $M / L$ ratio has increased by at least a factor of 1.6 from the center.

NGC 6703 , an asymptotically constant halo circular velocity $v_{0}$ can be determined with an accuracy of $\pm \lesssim 50 \mathrm{~km} \mathrm{~s}^{-1}$, at $95 \%$ c.l.

\section{Results}

For NGC 6703 we thus determine the true circular velocity at $2.6 R_{\mathrm{e}}$ to be $250 \pm 40 \mathrm{~km} \mathrm{~s}^{-1}$ (95\% c.l.), corresponding to a total mass inside $78^{\prime \prime}=$ $13.5 h_{50}^{-1} \mathrm{kpc}$ of $1.6-2.6 \times 10^{11} h_{50}^{-1} M_{\odot}$. No model without dark matter fits the data; however, a maximum stellar mass model in which the luminous component provides nearly all the mass in the centre does. In such a model, the total luminous mass inside $78^{\prime \prime}$ is $9 \times 10^{10} M_{\odot}$ and the integrated Bband $\mathrm{M} / \mathrm{L}$ out to this radius is $M / L_{\mathrm{B}}=5.3-10$ (95\% c.l.), rising from the central $M / L_{\mathrm{B}}=3.3$ by at least a factor of 1.6 .

The anisotropy of the stellar orbits in NGC 6703 changes from nearisotropic at the centre to radially anisotropic $\left(\beta=0.3-0.4\right.$ at $30^{\prime \prime}, \beta=$ $0.2-0.4$ at $\left.60^{\prime \prime}\right)$ and is not well-constrained at the outer edge of the data, where $\beta=-0.5-+0.5$, depending on variations of the potential in the allowed range.

\section{References}

Gerhard O.E. 1993, MNRAS, 265, 213

Merritt D. 1993, ApJ, 413, 79

Gerhard O.E., Jeske G., Saglia R.P., Bender R. 1998, MNRAS, in press

Saglia, R.P., Bender, R., Gerhard, O.E., Jeske, G. 1997, in Dark and Visible Matter in Galaxies and Cosmological Implications, Eds. M. Persic \& P. Salucci, ASP, 117, 113 